\title{
Eyewitness recognition errors: The effects of mugshot viewing and choosing in young and old adults
}

\author{
AMINA MEMON and LORRAINE HOPE \\ University of Aberdeen, Old Aberdeen, Scotland \\ JAMES BARTLETT \\ University of Texas at Dallas, Richardson, Texas \\ and \\ RAY BULL \\ University of Portsmouth, Portsmouth, England
}

\begin{abstract}
Eyewitness memory is vulnerable to information encountered prior to a lineup. Young (18-30 years) and older (60-80 years) witnesses viewed a crime video. Some witnesses were then exposed to mugshots of innocent suspects that included a critical foil. After a 48-h delay, all the witnesses took part in a targetabsent lineup that included the critical foil and five new foils. Witnesses who picked one of the mugshots as the likely perpetrator showed inflated rates of choosing the critical foil from the lineup. Context reinstatement instructions did not reduce choices of innocent foils following mugshot exposure. Despite age-related increases in false choosing, age did not qualify other effects. The results are discussed in terms of commitment, source memory, and gist-based processing.
\end{abstract}

Eyewitness testimony constitutes a very powerful form of evidence and can result in severe sentencing outcomes. One such example is the execution of a convicted murderer, Gary Graham, in Texas in June 2000. His case relied primarily on eyewitness evidence, but only one of eight witnesses actually made a positive identification from a lineup. Most relevant to the present paper is that the identification of the suspect from a live parade occurred 1 day after the witness had seen the same suspect's face in a photo array. The trial outcome suggests that the jury concluded that the witness accurately identified the suspect as the perpetrator. The witness in this case might well have been accurate, and certainly we cannot comment on the veracity of the decision. However, it is inevitable that the police will routinely show witnesses mugshots. Therefore, it is important to identify any negative effects of this investigative procedure.

In the present research, we explored several factors that might determine the impact of incidental exposure to the face of an innocent suspect that subsequently appears in a lineup. We had two specific goals. The first was to deter-

This research was supported by a grant from the Economic and Social Research Council, U.K. Thanks to Elizabeth Loftus and to three anonymous reviewers for comments on an earlier draft of this manuscript. Correspondence concerning this article should be addressed to A. Memon, Department of Psychology, University of Aberdeen, Kings College, Old Aberdeen, Scotland AB24 2UB (e-mail: amemon@ abdn.ac.uk). mine whether contemporary models of memory-most notably, the source monitoring framework (SMF) and a gist hypothesis derived from fuzzy trace theory (FTT) can account for the kinds of errors that arise in eyewitness identification situations, particularly those in which a suspect's face has been viewed by the witness before a lineup task. A second goal was to extend prior research on agerelated differences in false recognition previously observed with a variety of stimuli (Jacoby \& Hay, 1998; Koutstaal \& Schacter, 1997; Koutstaal, Schacter, Galluccio, \& Stofer, 1999), including faces (Bartlett, Strater, \& Fulton, 1991; Searcy, Bartlett, \& Memon, 1999).

Research interest in the effects of prior exposure began with the work of Brown, Deffenbacher, and Sturgill (1977). In a study simulating an eyewitness scenario, Brown et al. asked confederates (targets) to pass around some papers in class. Two or 3 days later, they showed participants 12 mugshot photographs containing one of the target faces. In a photo identification parade for the target 4-5 days later, the participants were significantly more likely to choose a nontarget face that was shown among the mugshot photographs than a nontarget face that had not been seen before (henceforth referred to as the mugshot exposure effect). In fact, nontarget faces that had been shown among the mugshots were chosen just as often in the identification parade as were true target faces. Later studies also confirmed that viewing mugshots has a detrimental effect on identification accuracy in subsequent recognition tasks (Brigham \& Cairns, 1988; Davies, Shepherd, \& Ellis, 1979; 
Gorenstein \& Ellsworth, 1980). These classic studies are important demonstrations of the interfering effects of mugshots, but they do not support a specific explanation for why these effects occur.

In a more recent study of the mugshot exposure effect, Dysart, Lindsay, Hammond, and Dupuis (2001) addressed a specific explanation that they termed the commitment effect. The idea is straightforward: If a witness picks a face from a set of mugshots and this face reappears in a lineup task, the witness will tend to pick that face again, owing to a feeling of commitment to the prior choice. To test this account, Dysart et al. exposed participants to up to 600 mugshots. In the commitment condition, a mugshot photograph (target) that was selected with the highest degree of confidence was included in a perpetrator-absent lineup. The lineup produced for that witness was shown to a second participant, who saw but did not select the target from the set of mugshots (source confusion or transference condition). The commitment group made significantly more incorrect identifications of the target photos than did the transference group, as well as a control (no-exposure) group. These findings suggest that false choosing can sometimes be due to commitment. However, further research is needed to determine whether other mechanisms also are involved.

According to the SMF (Johnson, Hashtroudi, \& Lindsay, 1993), decisions about the origin of a memory (source attributions) can be made on the basis of a variety of factors. These factors include the qualitative characteristics of the memory (e.g., vividness), consistency with general knowledge, and plausibility, as well as situational factors (e.g., motivation, social context, and costs of making a mistake). Source attributions can be made automatically and/or after more deliberate conscious processing, but it is generally accepted that source judgments are based on more stringent decision criteria than are recognition judgments (Hicks \& Marsh, 1999; Johnson et al., 1993; Marsh, Landau, \& Hicks, 1996; Multhaup, 1995). ${ }^{1}$ The SMF can readily be applied to the eyewitness identification situation, in which both the cognitive agenda and the social context can influence eyewitness decision making. For example, the SMF has been used to account for transference effects whereby an individual (bystander) seen prior to a crime event is mistakenly identified as the perpetrator (e.g., Read, 1994; Ross, Ceci, Dunning, \& Toglia, 1994). A simple account of this effect draws on the concept of context-free familiarity information, as presented in the dual-process framework (Jacoby, 1991; Mandler, 1980), and is in line with predictions of the SMF. A witness may make a lineup decision simply on the basis of a face's producing a strong feeling of familiarity, without due consideration of the contexts or source of this feeling. With respect to the mugshot exposure effect, the prediction is that a face viewed previously within a mugshot test will likely feel familiar when viewed in a lineup context, evoking false recognition to the extent that context-free familiarity information is the basis for responding. To test this hy- pothesis in the present study, one foil (deemed the critical foil $[\mathrm{CF}])$ appeared among the mugshots and also in the lineup task.

In addition to the source confusion and commitment interpretations, a third explanation for the mugshot exposure effect is suggested by the gist hypothesis. ${ }^{2}$ According to this hypothesis, false memories occur as a result of retrieval of gist memories (general representations or semantic content) when verbatim memory (the recall of exact surface details) would be more appropriate for the task (e.g., Koutstaal \& Schacter, 1997; Reyna, 1995; Reyna \& Brainerd, 1995). Since the mugshots were chosen to resemble the target face, exposing these items will strengthen gist-level representation. In turn, a stronger gist-level representation should lead to higher choosing rates for all the foils appearing in the subsequent lineup task.

Further predictions of the source confusion and the gist hypotheses were examined by including a group of older witnesses. Several recent studies have reported an agerelated increase in false recognitions both in laboratory studies (e.g., Koutstaal \& Schacter, 1997; Koutstaal et al., 1999) and in the eyewitness context (Memon \& Bartlett, 2002; Searcy et al., 1999, 2000; Searcy, Bartlett, Memon, $\&$ Swanson, 2001). These findings have often been interpreted as an increase in familiarity-based responding that is concomitant with a decline in contextual recollection in normal aging. If this reasoning is valid, age-related increases in false choosing should be greater for a face that has been previously viewed in a mugshot task, as compared with other faces. That is, the mugshot exposure effect should be increased for older witnesses, as compared with young adults.

Age-related differences in false recognition might also be due to an increase in gist-based processing in old age (Brainerd \& Reyna, 2001; Koutstaal et al., 1999; LaVoie \& Faulkner, 2000; Tun, Wingfield, Rosen, \& Blanchard, 1998). If so, and if presentation of a mugshot task can strengthen the gist-level representation of a face from a crime video, a clear prediction follows: Age-related differences in false choosing should be larger in conditions in which mugshots are presented than in control conditions, and this effect should hold not only with CFs, but also with other foils (OFs). In summary, either an agerelated deficit in source memory or an age-related increase in gist-level processing would cause older persons to be more susceptible to the mugshot exposure effect. However, the source confusion account would predict an agerelated increase in choice of the $\mathrm{CF}$, whereas the gist hypothesis would predict an age-related increase in choices of all the foils.

The extent to which either the source confusion or the gist hypothesis is supported may depend on the degree of environmental support provided at retrieval. Craik (1986) has argued that environmental support will help seniors in memory tasks, particularly in situations requiring selfinitiated processing. Recognition tests have been typically construed as providing maximal environmental support 
for retrieval (Craik, 1986). However, where many similar items are encountered on the test and where lures share many similarities with targets, still higher levels of environmental support might be required by seniors and, in some cases, by young adults as well (see Koutstaal \& Schacter, 2002, for a review). Instructions designed to facilitate recall of original contextual cues can, on occasion, reduce false choosing in eyewitness tasks (Cutler, Penrod, \& Martens, 1997; Gwyer \& Clifford, 1997; Kraffka \& Penrod, 1985; Malpass \& Devine, 1981; Smith \& Vela, 1992), although several studies report null effects (e.g., Fisher, Quigley, Brock, Chin, \& Cutler, 1990; Searcy et al., 2001). In the present study, we manipulated environmental support by comparing a standard lineup condition with one in which participants were encouraged to recollect context and to base recollection on what they had seen in the video.

In the present study, we investigated how false identifications in a lineup task might depend on (1) the viewing of mugshots prior to the lineup and (2) age. After viewing the crime event, old and young adults in the mugshot condition were exposed to a series of mugshots and were asked to judge whether one belonged to the perpetrator of the crime, although in fact, none of the mugshot faces belonged to the perpetrator. One of them, the CF, was repeated in a lineup given 2 days later. The design enabled us to look at the effects of preexposing a lineup face in a prior mugshot task (the mugshot exposure effect), as well as the effects of actually picking this foil in the prior task (i.e., the commitment effect).

In sum, in the present research, we focused on three theoretical perspectives in an attempt to understand mugshot exposure effects. The commitment hypothesis predicts that inflated false choosing rates occur for those witnesses who remain committed in a lineup task to a face selected in a prior mugshot task. In contrast, the source confusion account predicts that the mugshot exposure effect will result in increased choices of a CF that appears in both mugshot and lineup tasks, regardless of whether or not participants selected this or an OF in the mugshot task. A third perspective is the gist hypothesis, highlighted by FTT. On the assumption that mughots will strengthen gist, this view implies that mugshot exposure will increase false alarms to the CF (the foil that presented in both mugshot and lineup tasks) and to OFs as well. Hence, mugshot viewing will increase false alarms in response to all the faces in a target-absent lineup. The patterns predicted by the source confusion account and the gist hypothesis might be stronger among seniors than among young adults, although this might depend on environmental support.

\section{METHOD}

\section{Participants}

A total of 169 participants were tested, of whom 84 were students recruited within the University of Aberdeen and were between 18 and 30 years of age $(M=22.3$ years, $S D=3.0)$. The 85 older participants were recruited from the local community and were between 60 and 80 years of age ( $M=69$ years, $S D=6.2)$. All the participants were paid for contributing to the study.

\section{Design}

In a between-subjects design, younger and older adults were allocated to one of three groups. After viewing the event, the participants in the mugshot exposure (ME) and mugshot exposure plus context (MEC) conditions took part in a prelineup task that involved viewing a selection of mugshots. The third group served as a control and did not take part in this mugshot task after viewing the video event. All the participants viewed a target-absent lineup $48 \mathrm{~h}$ later.

\section{Materials}

Event. The witnessed event consisted of a video clip of a brief crime reconstruction in the form of a news report. This event was produced for Crimestoppers (a cable television program in the U.S.) and involved professional actors and production crews. The reconstruction involved the theft of a sports car from a car showroom. The clip shows two men entering a car showroom and asking to take a particular car for a test drive. The showroom salesman agrees and leaves the showroom in the car with one of the men. During the test drive, the car suddenly pulls over; the "customer" threatens the salesman with a gun and tells him to get out of the car. The car is then shown pulling away as the report comes to an end. The reporter finishes by saying that eyewitnesses identified the suspects as two white men. No other details about the suspects are provided in the reporter's narrative. The entire sequence lasts approximately $1 \mathrm{~min}$. The target's face was directly visible for $26 \mathrm{sec}$.

Mugbook. Two target-absent mugbooks were prepared. Each book consisted of 12 black-and-white $4 \times 3$ in. photographs of white males (head and shoulders only) of an age similar to that of the main target depicted in the event. Our mugbooks modeled the witness book format used in Scotland, where instead of asking the witness to search through a large database of faces, the police frequently select a smaller number of faces of suspects who have previously committed crimes that fit the category under investigation. In line with the target, none of these individuals wore facial hair or spectacles, and all had short, medium to dark hair. Only 1 photograph appeared on each page, and each page was numbered sequentially. The mid-booklet position (Photograph 6) was occupied by the CF. This was an individual who appeared both in the mugbook and in the lineup but who did not appear in the actual event. Two mugbooks were generated in which the $\mathrm{CF}$ differed, but the 11 other foils remained identical. Approximately half the participants in the mugshot exposure conditions saw one mugbook, whereas the remainder saw the other. An alternative mugbook was produced to ensure that any effects obtained with the mugshot exposure manipulation could not be attributable to any idiosyncratic features of a single $\mathrm{CF}$. The faces in the mugbook were selected in the same way as the faces in the lineup. We used a combination of the match-to-target and match-to-description strategies (see Tunnicliff \& Clark, 2000, for a review of foil selection procedures). The mugbook and lineup foils were selected from a larger pool of about 40 faces on the basis of their similarity ratings to the target photographs. These ratings were obtained from independent judges in a pilot study. The individual foils (including both CFs) did not differ significantly from each other in their similarity ratings to the target. All the foils selected fitted the general description of the target.

Lineup. The lineup consisted of six large $(10 \times 8$ in.) colored photographs presented in a $3 \times 2$ array. Thus, the lineup task differed considerably from the mugshot task, in that it involved a simultaneous array of a relatively small number of color photographs (adopting the conventions of lineup procedures in Scotland, where an identification parade generally consists of a suspect and between five and eight foils).

Screening measures. Before proceeding to the main testing session, the older sample completed two age-related screening measures. One such measure was the Mini Mental State Examination (MMSE; Folstein, Folstein, \& McHugh, 1975). This is an evaluative tool frequently used by clinicians to assess cognitive functioning in patients. The shortened version comprises 19 questions and takes approximately 5-10 min to complete. The average MMSE score ob- 
tained from the older adult sample in this study was 29.8 (maximum score $=30$ ), indicating intact cognitive function. The Geriatric Depression Scale (GDS) was also administered to older participants in order to screen for depression. The GDS, essentially a mood assessment scale, has been shown to elicit accurate ratings of minor, major, and no depression states, based on clinical diagnoses and symptom checklists (Brink et al., 1982). The average GDS score obtained from the present sample was 5.8, indicating the absence of severe depressive conditions within this particular sample of older adults. In fact, no older adult scored highly on the GDS, and all the scores were well below the clinical cutoff point for depression. The younger and older participants underwent a brief vision test using the Snellen eyechart. Any participants wearing corrective lenses were also required to take this vision test. The purpose of the test was to exclude any participants whose vision in their best eye was less than 20/50. No participants fell into this category.

Procedure. In the first testing session, the participants were seated in front of a wall-mounted screen onto which the video event was projected. Following the video event, the participants completed a number of filler tasks until $20 \mathrm{~min}$ had lapsed. The first test session came to a close for control participants at this point. The ME and MEC groups were then asked to read a set of written instructions requiring them to look carefully at the photographs in the mugbook with a view to identifying the armed car thief they had seen in the earlier video event. The total number of photographs contained in the mugbook was not disclosed at the outset. It was made clear that the perpetrator might or might not be among the photographs.

After viewing all 12 photographs, the participants were required to indicate whether or not they believed the target appeared in the mugbook and, if so, in which photograph. Confidence ratings on a 7-point scale for this decision were also obtained. Following a delay of $48 \mathrm{~h}$, the second testing session began with a target-absent lineup in which one foil (the $\mathrm{CF}$ ) was previously encountered in the mugbook task. Prior to the lineup, the participants were required to indicate how confident they were (on a 7-point scale) that they could identify the target seen in the video 2 days previously. The experimenter emphasized that the target might or might not be present in the lineup. Prior to viewing the lineup, the participants in the MEC condition were supplied with the following written instructions:

I am going to show you a lineup of photographs that may or may not contain the car thief from the video. Before I do that I would like you to think back to the video. Try and get a picture in your head of what you saw in the video. Start with when you first came into the room and sat down facing the screen. The opening sequence of the video showed a couple of men entering a car showroom.

Now, go through the video in your head and see if you can come up with what the car thief looked like. It is important that you base your decision on what you remember from seeing the video only and not any photographs that you looked at afterwards. Please focus on what you saw on the video and nothing else.

The participants read the instructions carefully and were given ample time to reinstate context. To ensure that the instructions were understood and followed, they were reiterated by the experimenter, and the witness was given additional time to reinstate context before proceeding to the lineup task. Once a rejection or identification decision had been made, the participants were asked to indicate their confidence in the accuracy of their identification decision on a 1- to 7-point scale.

\section{RESULTS}

We begin with an analysis of the mugshot-choosing data, followed by an analysis of the effects of age and ME condition on lineup decisions. We then turn to the relationship between the participants' responses in the mugshot task and their decisions in the lineup task.

\section{Mugbook Choosing}

Recall that there were two mugbooks containing two different $\mathrm{CFs}$, to eliminate the possibility that any effects of mugshot exposure were restricted to a particular face. Chi-square analysis indicated that (1) there were no significant differences in rates of overall mugshot choosing between the two mugbooks $\left[\chi^{2}(1, N=80)<1\right.$ n.s.], (2) the frequency with which each of the CFs was selected from the mugbooks did not differ significantly $\left[\chi^{2}(1, N=\right.$ $80)<1$ n.s.], and (3) there were no significant differences in the confidence with which mugshot choices were made between the mugbooks $(F<1)$. Therefore, the data for both mugbooks were pooled. In terms of overall mugshot choosing rates, $57 \%$ of those exposed to mugshots in the prelineup task chose a foil from the mugbook selection. The false choosing rates (collapsed across conditions) for the young and the older adults who were given the mugshot identification task were $42 \%$ and $71 \%$, respectively $\left[\chi^{2}(1, N=129)=10.72, p<.01\right]$.

\section{Lineup choosing}

The participants could produce either a correct rejection (CR) of the lineup or one of two types of false identification: a CF identification or an OF identification. Table 1 displays these three measures for young adults and seniors in each of the three conditions of the experiment. These data were subjected to hierarchical loglinear analysis (HILOG), which included the variables of age, condition, and decision (CF vs. OF vs. CR). The best-fitting model $^{3}$ included age $\times$ decision and condition $\times$ decision effects and provided an adequate fit $\left[\chi^{2}(6, N=169)=\right.$

Table 1

Proportions of Young and Senior Adults Making Critical Foil Choices, Other Foil Choices, and Correct Lineup Rejections in the Mugshot Exposure (ME), Mugshot Exposure Plus Context (MEC), and Control Conditions

\begin{tabular}{lccccccc}
\hline & \multicolumn{3}{c}{ Young Adults } & & \multicolumn{3}{c}{ Senior Adults } \\
\cline { 2 - 3 } \multicolumn{1}{c}{ Condition } & $\begin{array}{c}\text { Critical Foil } \\
\text { Choice }\end{array}$ & $\begin{array}{c}\text { Other Foil } \\
\text { Choice }\end{array}$ & $\begin{array}{c}\text { Correct } \\
\text { Rejection }\end{array}$ & & $\begin{array}{c}\text { Critical Foil } \\
\text { Choice }\end{array}$ & $\begin{array}{c}\text { Other Foil } \\
\text { Choice }\end{array}$ & $\begin{array}{c}\text { Correct } \\
\text { Rejection }\end{array}$ \\
\hline ME & .30 & .11 & .59 & & .35 & .42 & .23 \\
MEC & .25 & .05 & .70 & & .23 & .23 & .54 \\
Control & .20 & .00 & .80 & & .05 & .45 & .50 \\
All participants & .26 & .07 & .67 & & .25 & .38 & .38 \\
\hline
\end{tabular}


$7.99, p=.24]$. The age $\times$ decision effect $\left[\chi^{2}(2, N=\right.$ $169)=27.40, p<.01]$ reflected the fact that the young adults, as compared with the older adults, made more CRs $(M$ proportions $=.67$ and .38 , respectively $)$ and fewer (erroneous) $\mathrm{OF}$ choices $(M \mathrm{~s}=.07$ and .38 , respectively). The CF choices were about equally frequent in the two age groups ( $M \mathrm{~s}=.26$ and .25 , respectively). In line with this characterization of the data, follow-up HILOGs supported the interaction of age with the $\mathrm{OF}\left[\chi^{2}(1, N=169)=23.2\right.$, $p<.01]$ and the $\mathrm{CR}\left[\chi^{2}(1, N=169)=15.70, p<.01\right]$ measures, but not with the CF measures $\left[\chi^{2}(1, N=169)\right.$ $<1$, n.s.].

The condition $\times$ decision effect $\left[\chi^{2}(4, N=169)=\right.$ $12.0, p=.02]$ reflected the fact that $\mathrm{CF}$ choices fell from the ME condition to the MEC condition to the control condition ( $M$ proportions $=.32, .24$, and .12 , respectively), whereas CRs were lower in the ME condition than in the MEC and control conditions ( $M$ proportions $=.41, .62$, and .65 , respectively). OF choices showed no clear pattern $(M$ proportions $=.26, .14$, and .22 , respectively). Again, this characterization was supported by follow-up HILOGs: The condition effect was reliable with both the $\mathrm{CF}\left[\chi^{2}(2, N=169)=6.13, p=.05\right]$ and the $\mathrm{CR}\left[\chi^{2}(2\right.$, $N=169)=9.60, p<.01]$ measures, but not with the $\mathrm{OF}$ measure $\left[\chi^{2}(2, N=169)=3.45, p=.18\right]$.

In summary, whereas both age and condition affected CRs, the two variables differed with respect to the two different types of false choice error. Age affected primarily OF errors, whereas condition affected primarily $\mathrm{CF}$ errors. Although there is some suggestion in Table 1 that the mugshot exposure effects were increased among seniors, the HILOG did not support the age $\times$ condition $\times$ decision interaction $\left[\chi^{2}(4, N=169)=6.64, p=.16\right]$.

\section{Mugshot Choosing and Lineup Performance}

One of our goals was to determine whether the commitment hypothesis of Dysart et al. (2001) would provide a complete account of the inflated choosing rates that were due to mugshot preexposure. Had these inflated falsechoosing rates been due solely to commitment, they should have been found only among those witnesses who chose CFs in a prior mugshot task. Our first step in assessing this possibility was to compare mugshot choosers and mugshot nonchoosers in the lineup task. We classified each participant in the ME and MEC conditions as either a chooser or a nonchooser on the basis of their mugshot responses and conducted an additional HILOG analysis comparing three groups: mugshot choosers, mugshot nonchoosers, and controls (who were not exposed to mugshots). Other variables in the analysis were age and lineup decision (CF, OF, and CR). The best-fitting model included an age $X$ lineup decision effect and a lineup decision $X$ group interaction that showed adequate fit $\left[\chi^{2}(4, N=\right.$ $169)=7.19, p=.12]$. The age $\times$ lineup decision effect $\left[\chi^{2}(2, N=169)=24.4, p<.01\right]$ was described earlier: Older adults made more mugshot choices and more OF choices (and fewer CRs) in the lineup test. The group $\times$ lineup decision effect $\left[\chi^{2}(2, N=169)=16.05, p<.01\right]$ is new. As is shown in Table 2, mugshot choosers made more $\mathrm{CF}$ choices and fewer CRs, as compared with nonchoosers and controls $(M \mathrm{~s}=.40, .16$, and .13 , respectively, for CFs and .36, .64, and .65, respectively, for CRs). However, the choosers, nonchoosers, and controls showed quite similar rates of $\mathrm{OF}$ choices $(M \mathrm{~s}=.25, .20$, and .23 , respectively). Follow-up HILOGs confirmed reliable differences in lineup choices among choosers and nonchoosers $\left[\chi^{2}(2, N=129)=9.58, p<.01\right]$ and choosers and controls $\left[\chi^{2}(2, N=113)=11.43, p<.01\right]$. There was no reliable difference between nonchoosers and controls in the pattern of lineup choices $\left[\chi^{2}(2, N=94)=\right.$ 0.21, n.s.; see Table 2].

At first blush, Table 2 appears to be consistent with the notion that commitment might provide a complete explanation for the inflated rates of $\mathrm{CF}$ choosing in lineup tasks. However, only 13 of the 73 mugshot choosers (18\%) actually chose the CF in the mugshot task. Thus, most mugshot choosers had not shown commitment to the CF item that appeared in the lineup. To directly test the commitment hypothesis, after dropping the 13 participants who originally chose the $\mathrm{CF}$, we recomputed the proportion of $\mathrm{CF}$, OF, and $\mathrm{CR}$ decisions made by the mugshot choosers. As is shown in Table 2 (see the values in the last row), the pattern of results was unchanged. A new HILOG analysis showed again a significant effect of group (mugshot choosers, nonchoosers, and controls) on lineup decision, $\left[\chi^{2}(4, N=\right.$ $156)=10.31, p=.03]$. Mugshot choosers differed from nonchoosers $\left[\chi^{2}(2, N=116)=5.99, p=.05\right]$, and controls also differed from choosers $\left[\chi^{2}(2, N=100)=7.80\right.$, $p<.05]$. In conclusion, although the mugshot exposure effect was linked to choosing in the mugshot task, it did not depend, at least not entirely, on prior commitment to the CF.

We do not claim that commitment plays no role whatsoever in false identifications of CFs within the lineup task. Indeed, the 13 mugshot choosers who had picked the CF were especially likely to identify this same face within the lineup task $(.61 \%)$. The remaining (60) mugshot choosers who had picked someone else in the mugshot task picked the $\mathrm{CF}$ at a somewhat lower (albeit still inflated) rate in the subsequent lineup task (.35\%). The difference between $\mathrm{CF}$ choosers and other mugshot foil choosers approached reliability $\left[\chi^{2}(1, N=60)=3.14\right.$,

Table 2

Proportions of Critical Foil (CF) Choices, Other Foil (OF) Choices, and Correct Rejections (CRs) for Mugshot Choosing for Mugshot Exposure and Mugshot Exposure Plus Context Participants and for Control Participants (Who Did Not See Mugshots)

\begin{tabular}{lccc}
\hline \multicolumn{1}{c}{ Group } & CF & OF & CR \\
\hline Control & .13 & .23 & .65 \\
Mugshot nonchoosers & .16 & .20 & .64 \\
Mugshot choosers & .40 & .25 & .36 \\
Mugshot choosers (minus 13) & .35 & .25 & .40 \\
\hline
\end{tabular}

Note-The data in the last row are the CFs, OFs, and CRs for the mugshot choosers when the 13 participants who chose the CF in the mugshot phase were excluded. 
$p=.08]$, and this might suggest that commitment played a partial role in producing CF choices. In sum, our findings suggest that the effects of mugshot exposure on $\mathrm{CF}$ choices were carried largely by the mugshot choosers, including those choosers who picked the $\mathrm{CF}$, as well as those choosers who picked someone else. However, an alternative view of the data must be addressed: Perhaps mugshot choosing reflects a lenient criterion for recognition. That is, selecting a face in the mugshot task might reflect the use of a lax criterion that also promotes choice in the subsequent lineup. A problem for this notion is posed by a finding that we mentioned previously: Mugshot choosers exceeded mugshot nonchoosers in CF choices, but not in OF choices, in the lineup task (see Table 2). Thus, mugshot choosing was linked specifically to choices of the CF as opposed to the OFs. To further test this observation, we conducted one additional analysis. We included only those participants who made a lineup choice (CF vs. OF). Thus, the lineup decision variable had only two levels ( $\mathrm{CF}$ and $\mathrm{OF}$ ). To compensate for the loss of statistical power, we collapsed across the control and mugshot nonchooser groups to create a combined nonchooser group to compare with mugshot choosers. (Note that the control and the mugshot nonchooser groups showed virtually identical data in Table 2.) The analysis supported the critical group $\times$ decision interaction $\left[\chi^{2}(1, N=81)=7.31, p<.01\right]$, reflecting the fact that the mugshot choosers who made a lineup choice picked the $\mathrm{CF}$ at a rate of $62 \%$, whereas the mugshot nonchoosers and controls who made a lineup choice picked the $\mathrm{CF}$ at a rate of only $41 \%$.

\section{Confidence and Accuracy}

A measure of confidence was taken both before and after the lineup task. Confidence was measured on a scale of 1 to 7, where a high score indicated a high degree of confidence in the ability to identify the correct person if he were present in the lineup (prelineup) and in the decision made in the lineup task (postlineup). There was a significant correlation between pre- and postlineup confidence $[r(81)=.35, p<.01]$. There were no significant age differences in preconfidence ratings $[M s$ for young and old $=4.47$ and $4.14 ; F(1,167)=2.52, p>.05]$. However, the younger adults were significantly more confident than the older adults after the identification task [postconfidence; $M \mathrm{~s}=4.85$ and $4.17 ; F(1,164)=7.48, p<$ $.01]$. Given the notable age differences in lineup performance, we looked at the confidence-accuracy relationships separately for the young and the older participants. For the young participants, prelineup confidence was related to accuracy $[r(81)=.25, p<.05]$, as was postlineup confidence $[r(81)=.38, p<.01]$. There were no significant correlations for the older participants.

\section{DISCUSSION}

In the present research, we set out to identify the mechanisms responsible for false choosing in a lineup identification task following exposure to a set of mugshot photographs. We expected to replicate earlier findings (e.g.,
Brown et al., 1977) that a foil was chosen more often if it had been seen previously in a mugshot task than if it had not been seen before. In line with predictions, an exposure of an innocent foil prior to a lineup increased the rate of false choices of that foil. Most noteworthy among our new findings is that those witnesses who made any selection from the target-absent mugbook task were more likely to make a false choice of the familiarized $\mathrm{CF}$ than were those witnesses who made no mugshot choice. Indeed, the witnesses who saw the mugshots but made no selection showed no greater tendency to choose the CF in the final lineup than did the control witnesses, who saw no mugshots at all. The finding is reminiscent of Dysart et al. (2001), who found in their study that the mugshot effect appeared only for witnesses who picked a mugshot and chose that very face again in the lineup task. However, unlike Dysart et al., we found a clear mugshot effect for the majority of witnesses $(82 \%)$ who originally chose a mugshot face other than that which subsequently served as the CF in the lineup. Thus, the participants' prior commitment to the $\mathrm{CF}$ (as indicated by choosing that foil) was not a necessary prerequisite for the mugshot effect. Yet, making some mugshot choice, as opposed to viewing the mugshots but not choosing any, was a prerequisite for observing the effect. This effect is intriguing because it is counter to intuition. One might have predicted that a witness who has chosen a mugshot face and then is presented with a lineup that does not include that face would be very unlikely to make a choice. After all, the face he or she recognized previously as the culprit simply is not there. Yet we found that mugshot choosers were highly likely to make lineup choices whether or not a previously chosen face was present.

It might be argued that mugshot choosing was related to lineup performance because it indicated a lax criterion for identification. However, the effect was restricted to false identifications of the familiarized foil. False identifications of OFs were not related to the mugshot choice variable (see Table 2). A more plausible account holds that the mugshot choosing indicated a particular strategy for making recognition judgments. Specifically, we suggest that mugshot choosers used perceived familiarity as a basis for choosing in both the mugshot task and the subsequent lineup. By contrast, mugshot nonchoosers were more likely to use conscious recollection of perceptual and contextual information as a basis for their responses. Owing to the fact that mugshots were chosen to resemble the target face, one or more of these faces was likely to be perceived as familiar by any given participant. Hence, if a participant were using perceived familiarity as a basis for a response, he or she would be likely to identify a mugshot face. It also is likely that the CF in the lineup task was perceived as familiar (in the mugshot condition), owing to its prior presentation. For this reason, a witness in the mugshot condition who used a familiarity strategy would be highly prone to choose the critical lineup foil, indeed more likely to choose this $\mathrm{CF}$ than to choose other lineup foils.

This familiarity strategy hypothesis is consistent with the finding that our older participants made more choices in both the mugshot task and the subsequent lineup. These 
age differences in false choosing are in line with much evidence that seniors, as compared with young adults, make more use of perceived familiarity, as opposed to recollection, in the recognition of faces (Bartlett \& Fulton, 1991; Bartlett et al., 1991; Searcy et al., 1999) and other stimuli (Dywan \& Jacoby, 1990; Jennings \& Jacoby, 1997; Parkin \& Walter, 1992). Another finding consistent with the familiarity-strategy hypothesis is the lack of reliable age differences in lineup performance when mugshot choosing was taken into account in our conditionalized analyses. Presumably, the conditionalized scores served to partial out effects linked to use or nonuse of the familiarity strategy. If age differences in performance are linked to the use of a familiarity versus a recollection strategy, no age differences would be expected with the conditionalized scores.

How do the source confusion and gist hypotheses fare with the present data? The source confusion hypothesis correctly predicted that the mugshot exposure effect would be restricted to the familiarized $\mathrm{CFs}$, as opposed to the OFs. However, the source confusion hypothesis, as was stated in the introduction, also predicted that the mugshot exposure effect would be increased in old age. Although there were trends in line with this prediction (see Table 1, columns 1 and 4), they were not supported statistically. In addition, the source confusion account would not have predicted that the mugshot exposure effect would depend on mugshot choosing. We therefore suggest that the source confusion account be modified as follows: Source confusion errors in the lineup task reflect the use of a familiarity strategy that also produces high rates of mugshot choosing. It appears that this strategy is used more by older witnesses (see Bartlett et al., 1991; Searcy et al., 1999), although the present research is somewhat ambiguous on the latter point.

The gist hypothesis fares rather more poorly. That view predicted that mugshot exposure would increase false choosing not only of familiarized foils, but also of OFs as well. That the effect was restricted to familiarized foils (Table 1) contradicts the hypothesis. Moreover, although the gist hypothesis correctly predicted the age-related increase in OF choices (Table 1, columns 2 and 5), it incorrectly predicted that the size of this increase would be greater in the mugshot exposure condition than in the control condition. There was no such trend in the data (see Table 1, columns 2 and 5).

The notion of gist is strongly associated with the fuzzy trace framework of Brainard, Reyna, and colleagues (Brainerd \& Reyna, 1990). However, although our data pose problems for a gist interpretation of the mugshot exposure effect, they should not be viewed as disconfirming the broader fuzzy trace model. By current formulations of the fuzzy trace model, false memories occur in two ways. First, they occur as a result of retrieval of gist memories (general representations or semantic content) when verbatim memory (the recall of exact surface details) would be more appropriate for the task. This is essentially what we have been calling the gist hypothesis in the present paper. Second, false memories occur at the level of verbatim memory when the context of the verbatim trace is erroneously retrieved (Reyna \& Lloyd, 1997). Thus, mugshot exposure might produce a verbatim memory for a mugshot face that might lead to a subsequent false identification of that face if the witness retrieves the context of the crime instead of the context of the mugshot task. Witnesses who choose in the mugshot task might differ from witnesses who do not choose in being more subject to context retrieval errors. This would explain why mugshot choosing is linked to the mugshot exposure effect. Finally, older witnesses might be more subject to context retrieval errors. This would explain the age-related increases in false choosing that were observed in this study.

The verbatim context error account strongly resembles the source memory account we have favored in this paper. Further research clearly is needed to distinguish these quite similar ideas. Further research is also needed to explore other implications of the fuzzy trace framework in lineup identification tasks. In particular, whether exposure to a small number of faces is sufficient to create an effective gist representation has yet to be addressed.

Since our focus in this study was on the mechanisms responsible for false choosing in lineups (cf. Searcy et al., 2000; Wells \& Luus, 1990), we did not include a targetpresent condition in which the true perpetrator would appear in the lineup. Because of this limitation, we do not know whether the negative effect of mugshot exposure (and mugshot choosing) might be balanced, in a sense, by a positive effect of viewing (and choosing) the true criminal as a mugshot before he or she appears in the formal lineup (cf. Brown et al., 1977). One question raised by these considerations is that of whether our mugshot exposure (and choosing) effects might reflect differences in response bias, as opposed to differences in true memory (discrimination).

Although we do not deny that target-present lineups can provide useful data, we have previously rejected a bias account on the basis of the finding that mugshot exposure and mugshot choosing affected only choices of familiarized foils. OF choices were not affected by these variables. In addition, our findings obtained with a target-absent lineup support two important conclusions about the effects of mugshot viewing on subsequent identification performance. First, the mugshot exposure effect with targetabsent lineups is linked to mugshot choosing: Only those witnesses who choose a mugshot face show the mugshot exposure effect. Second, the mugshot exposure effect with target-absent lineups is not simply a commitment effect: Thus, a witness who has viewed a set of mugshots and has selected one of them is at high risk of falsely identifying one of the mugshot faces. This is true even when the previously selected face is absent from the lineup.

Of course, if the previously selected face were to be present in the lineup, matters might be even worse. As was stated in the introduction, Dysart et al. (2001) found that a lineup face was more likely to be chosen if it had been viewed and selected in a prior mugshot task than if it had been viewed but not selected in that task. Here, we found 
a trend in the same direction, although it was not statistically reliable. Such evidence for a commitment effect might be stronger in some conditions than in others. We note that Dysart et al. presented up to 600 faces in their mugshot task. Memory for a face among so many mugshots would be expected to be poor unless that face were chosen as the perpetrator. By contrast, the mugshot task in the present study included only 12 faces, and so memory for even the nonchosen faces quite plausibly was strong. Perhaps the commitment effect occurs to the extent that a viewed and selected face is perceived as more familiar than a viewed but not selected face in the lineup task. This may have been true in the Dysart et al. study much more than in ours. In any case, the major applied implication of this study is clear: Although the occasional use of mugshot tasks might be unavoidable, a witness who selects a face in a mugshot task should not be subject to a formal lineup. Our results indicate that this is true even if the previously chosen face turns out to be that of an innocent suspect and is absent from the subsequent lineup.

In closing, we consider the extent to which context reinstatement may provide environmental support at retrieval particularly for seniors. This hypothesis was not supported. Although the context reinstatement instructions prior to a lineup brought the CR rate to a level comparable with a control group who was not exposed to mugshots, they had no reliable effect on CF choices. In other words, exposure to mugshots - and selection of one of them-can lead to false identifications in the lineup task, even when context reinstatement instructions are used for the lineup.

\section{REFERENCES}

Bartlett, J. C., \& Fulton, A. (1991). Familiarity and recognition of faces: The factor of age. Memory \& Cognition, 19, 229-238.

Bartlett, J. C., Strater, L., \& Fulton, A. (1991). False recency and false fame of faces in young adulthood and old age. Memory \& Cognition, 19, 177-188.

Brainerd, C. J., \& Reyna, V. F. (1990). Gist is the grist: Fuzzy trace theory and the new intuitionism. Developmental Review, 10, 365-403.

Brainerd, C. J., \& Reyna, V. F. (2001). Fuzzy trace theory: Dual processes in memory, reasoning and cognitive neuroscience. $A d$ vances in Child Development \& Behavior, 28, 41-100.

Brigham, J. C., \& Cairns, D. L. (1988). The effect of mugshot inspections on eyewitness identification accuracy. Journal of Applied Social Psychology, 18, 1394-1410.

Brink, T., Yesavage, J., Lum, O., Heersema, P., Adey, M., \& Rose, T. (1982). Screening tests for geriatric depression. Clinical Gerontologist, 1, 37-44.

Brown, E., Deffenbacher, K., \& Sturgill, W. (1977). Memory for faces and the circumstances of encounter. Journal of Applied Psychology, 62, 311-318.

Craik, F. I. M. (1986). A functional account of age differences in memory. In F. Klix \& H. Hagendorf (Eds.), Human memory and cognitive capabilities, mechanisms and performances (pp. 409-422). Amsterdam: Elsevier, North-Holland.

Cutler, B. L., Penrod, S. D., \& Martens, T. K. (1997). Improving the reliability of eyewitness testimony: Putting context into context. Journal of Applied Psychology, 72, 629-637.

Davies, G., Shepherd, J., \& Ellis, H. (1979). Effects of interpolated mugshot exposure on accuracy of eyewitness identification. Journal of Applied Psychology, 64, 232-237.

Dysart, J., Lindsay, R. C. L., Hammond, R., \& Dupuis, P. (2001). Mug shot exposure prior to lineup identification: Interference, transference and commitment effects. Journal of Applied Psychology, 86, 1280-1284. DYWAN, J., \& JACOBY, L. (1990). Effects of aging on source monitoring: Differences in susceptibility to false fame. Psychology \& Aging, 5, 379-387.

Fisher, R. P., Quigley, K. L., Brock, P., Chin, D., \& Cutler, B. L. (1990). The effectiveness of the cognitive interview in description and identification tasks. Paper presented at the biennial meeting of the American Psychology and Law Society, Williamsburg, VA.

Folstein, M. F., Folstein, S. E, \& McHugh, P. R. (1975). "Mini-mental state": A practical method for grading the cognitive state of patients for the clinician. Journal of Psychiatric Research, 12, 189-198.

Gorenstein, G. W., \& Ellsworth, P. C. (1980). Effect of choosing an incorrect photograph on a later identification by an eyewitness. Journal of Applied Psychology, 65, 616-622.

GWyer, P., \& Clifford, B. R. (1997). The effects of the cognitive interview on recall, identification, confidence and the confidence/ accuracy relationship. Applied Cognitive Psychology, 11, 121-145.

HicKs, J. L., \& MARSH, R. (1999). Attempts to reduce the incidence of false recall with source monitoring. Journal of Experimental Psychology: Learning, Memory, \& Cognition, 24, 1137-1151.

JACOBY, L. L. (1991). A process dissociation framework: Separating automatic from intentional uses of memory. Journal of Memory \& Language, 30, 513-541.

JACOBY, L. L., \& HAY, J. F. (1998). Age-related deficits in memory: Theory and application. In M. A. Conway, S. E. Gathercole, \& C. Cornoldi (Eds.), Theories of memory (Vol. II, pp. 111-134). Hove, U.K.: Psychology Press.

JENNINGS, J. M., \& JACOBY, L. L. (1997). An opposition procedure for detecting age related deficits in repetition: The telling effects of repetition. Psychology \& Aging, 12, 352-361.

Johnson, M. K., Hashtroudi, S., \& LindSay, D. S. (1993). Source monitoring. Psychological Bulletin, 114, 3-28.

Koutstaal, W., \& Schacter, D. L. (1997). Gist-based false recognition of pictures in older and younger adults. Journal of Memory \& Language, 37, 555-583.

Koutstaal, W., \& Schacter, D. L. (2002). Memory distortion and aging. In M. Naveh-Benjamin, M. Moscovitch, \& H. L. Roediger, III (Eds.), Perspectives on human memory and cognitive aging: Essays in honor of Fergus Craik (pp. 362-383). Philadelphia: Psychology Press.

Koutstaal, W., Schacter, D. L., Galluccio, L., \& Stofer, K. A. (1999). Reducing gist-based false recognition in older adults: Encoding and retrieval manipulations. Psychology \& Aging, 14, 220-237.

Kraffka, C., \& Penrod, S. (1985). Reinstatement of context in a field experiment on eyewitness identification. Journal of Personality \& Social Psychology, 70, 556-564.

La Voie, D. J., \& Faulkner, K. (2000). Age differences in false recognition using a forced choice paradigm. Experimental Aging Research, 26, 367-381.

LindSAY, D. S., \& Johnson, M. K. (2000). False memories and the source monitoring framework: Reply to Reyna and Lloyd (1997). Learning \& Individual Differences, 12, 145-161.

Malpass, R. S., \& Devine,P. G. (1981). Guided memory in eyewitness identification. Journal of Applied Psychology, 64, 343-350.

MANDLER, G. (1980). Recognizing: The judgment of previous occurrence. Psychological Review, 75, 421-441.

Marsh, R., LANDAU, J., \& Hicks, J. L. (1996). The post-information effect and reductions in retroactive interference. Journal of Experimental Psychology: Learning, Memory, \& Cognition, 22, 886-897.

Memon, A., \& Bartlett, J. C. (2002). The effects of verbalisation on face recognition. Applied Cognitive Psychology, 16, 635-650.

Multhaup, K. S. (1995). Aging, source, and decision criteria: When false fame errors do and do not occur. Psychology \& Aging, 10, 492-497.

Neuschatz, J. S., Payne, D. G., Lampinen, J. M., \& Toglia, M. P. (2001). Assessing the effectiveness of warnings and the phenomenological characteristics of false memories. Memory, 9, 53-71.

Parkin, A., \& Walter, B. (1992). Recollective experience, normal aging, and frontal dysfunction. Psychology \& Aging, 7, 290-298.

READ, J. D. (1994). Understanding bystander misidentifications: The role of familiarity and contextual knowledge. In D. F. Ross, J. D. Read, 
\& M. P. Toglia (Eds.), Adult eyewitness testimony (pp. 56-79). Cambridge: Cambridge University Press.

REYNA, V. F. (1995). Interference effects in memory and reasoning: A fuzzy trace theory analysis. In F. Dempster \& C. Brainerd (Eds.), Interference and inhibition in cognition (pp. 29-59). San Diego: Academic Press.

Reyna, V. F., \& Brainerd, C. J. (1995). Fuzzy-trace theory: An interim synthesis. Learning \& Individual Differences, 7, 1-75.

REYNA, V. F., \& LLOYD, F. (1997). Theories of false memory in children and adults. Learning \& Individual Differences, 9, 95-123.

Ross, D. F., Ceci, S. J., Dunning, D., \& Toglia, M. P. (1994). Unconscious transference and mistaken identity: When a witness misidentifies a familiar but innocent person. Journal of Applied Psychology, 79, 918-930.

Searcy, J. H., Bartlett, J. C., \& Memon, A. (1999). Age differences in accuracy and choosing in eyewitness identification and face recognition. Memory \& Cognition, 27, 538-552.

Searcy, J. H., Bartlett, J. C., \& Memon, A. (2000). Relationship of availability, lineup conditions and individual differences to false identification by young and older eyewitnesses. Legal \& Criminological Psychology, 5, 219-236.

Searcy, J. H., Bartlett, J. C., Memon, A., \& Swanson, K. (2001). Aging and lineup performance at long retention intervals: Effects of metamemory and context reinstatement. Journal of Applied Psychology, 86, 207-214.

Smith, S. M., \& Vela, E. (1992). Environmental context-dependent eyewitness recognition. Applied Cognitive Psychology, 6, 125-139.

Tabachnick, B. G., \& Fiddell, L. S. (2001). Using multivariate statistics (4th ed.). New York: Harper Collins.

Thierry, K., Spence, M., \& Memon, A. (2000). A comparison between fuzzy-trace theory and source-monitoring theory: Evidence from an eyewitness suggestibility study. In K. Roberts \& M. Blades (Eds.), Children's source monitoring (pp. 171-196). Mahwah, NJ: Erlbaum.

Tun, P. A., Wingfield, A., Rosen, M. J., \& Blanchard, L. (1998). Response latencies for false memories: Gist-based processes in normal aging. Psychology \& Aging, 13, 230-241.
TunNicliff, J., \& Clark, S. (2000). Selecting foils for identification lineups: Matching suspects or descriptions? Law \& Human Behavior, 24, 231-258.

Wells, G., \& LuUs, C. (1990). Police lineups as experiments: Social methodology as a framework for properly conducted lineups. Personality \& Social Psychology Bulletin, 76, 765-791.

\section{NOTES}

1. In the source monitoring framework, more stringent criteria refer to criteria involving more discriminating or diagnostic types of information, not simply greater levels of perceived familiarity, as in signal detection theory.

2 . We accept that the gist and source confusion hypotheses are not mutually exclusive (Neuchatz, Payne, Lampinen, \& Toglia, 2001; Thierry, Spence, \& Memon, 2000), but there are important differences (Lindsay \& Johnson, 2000).

3. A fit is considered adequate in HILOG if $p$ is greater than .05 (Tabachnick \& Fiddell, 2001).

4. An anonymous reviewer suggested that although a single-criterion hypothesis cannot explain our data, a combined criterion and commitment hypothesis might do the job. The idea is that choosing in the mugshot task is linked to (1) commitment to the selected mugshot and (2) a bias to choose other faces as well. We are not sure this hypothesis is workable, but nonetheless we tested it by repeating the HILOG of CF and OF choices, dropping not only those witnesses who made CRs in the lineup, but also those witnesses who picked the CF in the mugshot test. The results were materially unchanged from those detailed above: CF versus OF choosers fell from $62 \%$ to $58 \%$ (as compared with $41 \%$ for nonchoosers). A new HILOG once again supported the chooser/nonchooser difference $\left[\chi^{2}(1, N=68)=5.02, p=.02\right]$.

(Manuscript received July 9, 2001; revision accepted for publication August 15, 2002.) 